\title{
Feasibility of Serum Pentosidine Level as a Potential Risk Factor for Osteoporotic Vertebral Compression Fracture
}

\author{
Dong-Hyuk Choi, Sang-Min Lee, Sung-An Lim, Yong-Soo Choi \\ Department of Orthopaedic Surgery, Kwangju Christian Hospital, Gwangju, Korea
}

\begin{abstract}
Study Design: Feasibility study.
Purpose: To evaluate the feasibility of using serum pentosidine level as a potential marker for osteoporotic vertebral compression fracture (OVCF).

Overview of Literature: A review of previous studies suggests a negative correlation between serum pentosidine concentration and bone strength. However, it is unclear whether serum pentosidine level might be a potential marker of OVCF in Koreans.

Methods: Forty patients who underwent bone mineral density examination were included in this study, and their serum pentosidine levels were prospectively analyzed. Serum pentosidine level was evaluated using enzyme-linked immunosorbent assay. Among all the patients, 11 with OVCF were assigned to the vertebral fracture group and 29 who did not have vertebral fracture were included in the non-fracture group. In addition, we used the Fracture Risk Assessment (FRAX) tool Korean version for assessing the 10-year probability of fracture.

Results: There was a statistically significant difference in the mean serum pentosidine level $(p=0.04)$ of the vertebral fracture group $(110.8 \mathrm{ng} / \mathrm{mL})$ and the non-fracture group $(64.3 \mathrm{ng} / \mathrm{mL})$. Logistic regression analyses showed that serum pentosidine was significantly associated with OVCF. The vertebral fracture group had significantly higher 10-year probability of major osteoporotic fracture as per FRAX than the non-fracture group. There was a positive correlation between pentosidine level and FRAX results $(r=0.35, p=0.02)$.

Conclusions: These results suggest that increased serum pentosidine level could be a potential marker for OVCF.
\end{abstract}

Keywords: Vertebral compression fracture; Osteoporosis; Pentosidine

\section{Introduction}

Osteoporosis is a skeletal disorder characterized by compromised bone strength, and bone strength is determined by bone density and bone quality [1]. The World Health Organization (WHO) has proposed that osteoporosis be defined as bone mineral density (BMD) 2.5 standard deviations (SD) or more below the mean value for young health women (T-score $\leq-2.5 \mathrm{SD}$ ) using dual-energy radiography absorptiometry (DXA). This definition has been accepted by most regulatory agencies worldwide. It is being used as a cutoff to limit treatment access $[2,3]$. In 2005, Simon [4] reported that osteoporosis is defined as a disease characterized by low bone mass and micro-

Received Nov 14, 2017; Revised Apr 17, 2018; Accepted May 8, 2018

Corresponding author: Yong-Soo Choi

Department of Orthopaedic Surgery, Kwangju Christian Hospital, 37 Yangnim-ro, Nam-gu, Gwangju 61661, Korea

Tel: +82-62-650-5060, Fax: +82-62-650-5066, E-mail: stemcellchoi@gmail.com 
architectural deterioration of the bone tissue, leading to enhanced bone fragility and a consequent increase in fracture risk.

Type I collagen is a bone component; therefore, bone strength may be affected by the nature of collagen fibers in the bone. Several reports have indicated that the properties of collagen, including its composition and cross-links, can influence bone strength [5-7]. To evaluate the relationship between collagen and bone properties, it is necessary to understand the process of collagen formation in the bone. Stabilization of newly formed collagen fibers is initially achieved by the formation of covalent cross-links between adjacent collagen molecules. Collagen cross-links can be divided into the following two types: (1) lysine hydroxylase and lysyl oxidase-controlled cross-links (enzymatic cross-links), and (2) glycation or oxidation-induced advanced glycation end (AGE) products cross-links (nonenzymatic cross-links) [8]. Enzymatic cross-links may improve bone strength [9]. However, it has been generally accepted that non-enzymatic cross-links deteriorate the biological and mechanical functions of the bone $[8,10]$. Pentosidine, a well-known AGE, has been considered a useful surrogate marker of AGE in the bone [8].

There was a negative correlation between the serum pentosidine concentration and bone strength [11-13]. However, it is unclear whether serum pentosidine level can be a potential marker for osteoporotic vertebral compression fracture (OVCF) in Koreans. The hypothesis of this study was that serum pentosidine level is higher in people with OVCF. Therefore, this study aimed to evaluate whether it is feasible to use serum pentosidine level as a potential marker for OVCF.

\section{Materials and Methods}

From March 2015 to December 2015, 40 patients who were admitted at our hospital because of degenerative spinal disorders, including OVCF, and agreed to participate in this study were included. Further, this study was approved by the Institutional Review Board at the Kwangju Christian Hospital (IRB approval no., KCHIRB-M-2014-067). And informed consent was obtained from patients. To identify osteoporosis-related fractures, a cutoff value of 50 years of age was used $[14,15]$. Patients aged $<50$ years were excluded. Serum pentosidine level is elevated in people with diabetes mellitus [8] or decreased kidney function [16]; therefore, such patients were excluded. Patients with any history of medication that might affect bone metabolism, including glucocorticoids, sex hormones, warfarin, and bisphosphonates, were also excluded [17].

All patients underwent BMD examination and biochemical markers measurements. BMD was examined using DXA as per the manufacturer's instructions (Explorer; Hologic Co., Bedford, MA, USA). Osteoporosis was diagnosed as per the WHO criteria. Vertebral fractures were diagnosed with plain radiological examination and magnetic resonance imaging.

Eleven of the patients with OVCF were assigned to the vertebral fracture group, and 29 who did not have vertebral fracture were included in the non-fracture group. The average age of the subjects (two men and nine women) in the vertebral fracture group was 76.5 years. All the patients in the vertebral fracture group were diagnosed with osteoporosis. The average age of the subjects (five men and 24 women) in the non-fracture group was 68.4 years. Of the 29 patients, 19 were diagnosed with osteoporosis. There were no significant differences in the sex, age, or BMD of the two groups (Table 1).

For biochemical measurements, the patients' fasting blood samples were obtained, and their fasting plasma glucose, glycosylated hemoglobin (HbA1c), blood urea nitrogen (BUN), serum creatinine, serum alkaline phos-

Table 1. Demographic data

\begin{tabular}{lccc} 
Characteristic & Vertebral fracture group & Non-fracture group & $p$-value \\
No. of patients & 11 & 29 & 0.94 \\
Gender & & & \\
Male & 2 & 5 & 0.09 \\
\hline Female & 9 & 68.4 & 0.23 \\
\hline Mean age (yr) & 76.5 & -2.4 & \\
\hline Bone mineral density (T-score) & -3.9 & & \\
\hline
\end{tabular}


phatase, and osteocalcin levels were measured. The results of these biochemical measurements were compared between the two groups. The serum pentosidine level was measured using a quantitative sandwich enzyme-linked immunosorbent assay (ELISA) kit (Human Pentosidine ELISA Kit; MyBioSource Inc., San Diego, CA, USA).

All the patients answered the questionnaire that enabled the calculation of the 10-year probability of fracture with BMD using the Korean version of Fracture Risk Assessment (FRAX) tool (https://www.shef.ac.uk/FRAX/ tool.jsp?lang=ko) to determine significant risk factors of OVCF [18]. Although the use of corticosteroids at the time of the fracture was excluded in materials, the FRAX tool was analyzed as a risk factor in patients who had taken it for more than three months during their lifetime, as specified in the FRAX system. Moreover, we analyzed the correlation between FRAX and the pentosidine level.

Statistical analyses were performed using IBM SPSS Statistics ver. 22.0 for Windows IBM (IBM Corp., Armonk, NY, USA). Sex, age, and osteoporosis status of the patients were compared using the chi-square test. Clinical results were analyzed using the Mann-Whitney $U$-test. The correlation between FRAX and serum pentosidine level was analyzed to determine the significance of their relationship. Logistic regression analyses were performed to examine the contribution of each factor to OVCF. A $p$-value $<0.05$ was considered statistically significant.

\section{Results}

The mean serum pentosidine level was $110.8 \mathrm{ng} / \mathrm{mL}$ in the vertebral fracture group and $64.3 \mathrm{ng} / \mathrm{mL}$ in the non-fracture group. There was a statistically significant difference in the mean serum pentosidine level between the vertebral fracture group and the non-fracture group $(p=0.04)$.

Logistic regression analyses showed that corticosteroids use (odds ratio [OR], 19.97; $p=0.03$ ), smoking (OR, 14.82; $p=0.01$ ), serum pentosidine (OR, $1.03 ; p=0.01$ ), body weight (OR, $0.94 ; p=0.01$ ), HbAlc (OR, $0.91 ; p=0.01$ ), and $\mathrm{BMD}(\mathrm{OR}, 0.58 ; p=0.01)$, were significantly associated with OVCF (Table 2).

However, there was no significant differences in the fasting blood glucose $(p=0.70), \mathrm{HbA1c}(p=0.70)$, blood creatinine $(p=0.24), \mathrm{BUN}(p=0.33)$, blood alkaline phosphatase $(p=0.17)$, or serum osteocalcin $(p=0.55)$ levels of the two groups (Table 3 ). The mean lumbar BMD was -3.9 in the fracture group and -2.4 in the non-fracture group
Table 2. Multivariate regression analysis for osteoporotic vertebral fracture

\begin{tabular}{lcc} 
Variable & $p$-value & Odds ratio \\
\hline Corticosteroid use (yes or no) & 0.03 & 19.97 \\
\hline Smoking (yes or no) & 0.01 & 14.82 \\
Creatinine (mg/dL) & 0.07 & 1.07 \\
\hline Pentosidine (ng/mL) & 0.01 & 1.03 \\
\hline Body weight (kg) & 0.01 & 0.94 \\
Glycosylated hemoglobin (\%) & 0.01 & 0.91 \\
\hline Osteocalcin (ng/mL) & 0.07 & 0.79 \\
Bone mineral density (T-score) & 0.01 & 0.58 \\
Age (yr) & 0.52 & 0.45
\end{tabular}

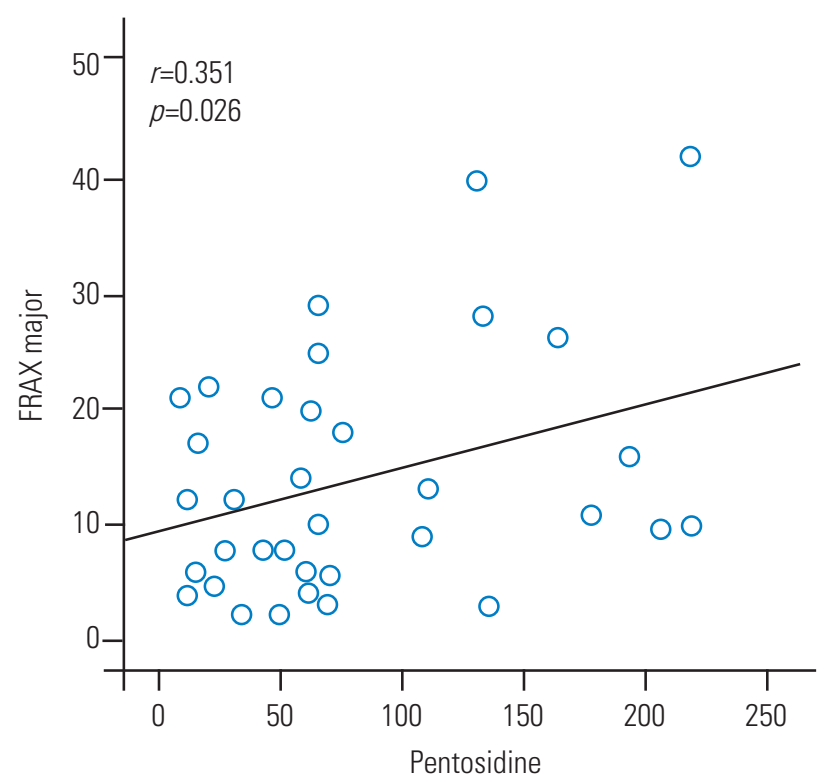

Fig. 1. Positive correlation between the serum pentosidine levels and FRAX results in major osteoporotic fracture. FRAX, Fracture Risk Assessment tool.

$(p=0.23)$ (Table 1).

The 10-year probability of vertebral fracture using FRAX was $19.1 \%$ in the vertebral fracture group and $10.8 \%$ in the non-fracture group. The vertebral fracture group had a significantly higher 10 -year probability of fracture than the non-fracture group $(p=0.05)$. Further, there was a positive correlation between the serum pentosidine level and the FRAX results $(r=0.35, p=0.02)$ (Fig. 1).

\section{Discussion}

Pentosidine is a well-known AGE discovered from aging 
Table 3. Comparison of biochemical results between the vertebral fracture group and the non-fracture group

\begin{tabular}{lccc} 
Variable & Vertebral fracture group & Non-fracture group & $p$-value \\
\hline Glycosylated hemoglobin (\%) & 6.0 & 5.9 & 0.70 \\
\hline Fasting blood glucose $(\mathrm{mg} / \mathrm{dL})$ & 125.7 & 123.0 & 0.70 \\
\hline Alkaline phosphatase $(\mathrm{U} / \mathrm{L})$ & 74.3 & 70.0 & 0.17 \\
\hline Blood urea nitrogen $(\mathrm{mg} / \mathrm{dL})$ & 13.1 & 15.0 & 0.33 \\
Creatinine $(\mathrm{mg} / \mathrm{dL})$ & 0.8 & 0.9 & 0.24 \\
Osteocalcin $(\mathrm{ng} / \mathrm{mL})$ & 16.3 & 18.0 & 0.55 \\
\hline Pentosidine $(\mathrm{ng} / \mathrm{mL})$ & 110.8 & 64.3 & 0.04 \\
\hline
\end{tabular}

Values are presented as mean results.

human extracellular matrix [19]. Pentosidine is accumulated in patients with low-turnover bone diseases, such as diabetes [20] or in those for whom bone resorption is suppressed with bisphosphonate [21] or the aging process [7]. To eliminate factors that might influence the serum pentosidine level in this study, patients with diabetes or on bisphosphonate medication were excluded. Furthermore, those with a history of medications that might affect bone metabolism were also excluded. Several studies have revealed that pentosidine content in the cortical or trabecular bone from the vertebra is negatively associated with the mechanical properties $[7,11,22]$. The serum pentosidine level is reported to have a significant linear correlation with pentosidine in the cortical bone [23] Pentosidine deteriorates the osteoblastic function [24]. Its accumulation makes the collagen fibers brittle and deteriorates the post-yield properties and toughness [25,26]. Our results suggest that increased serum pentosidine level could be a potential risk factor for OVCF.

The FRAX tool was developed by the WHO to evaluate the individualized 10-year probability of hip and major osteoporotic fracture [27]. The FRAX tool is based on individual patient models that integrate the risks associated with clinical risk factors and BMD at the femoral neck [28]. The FRAX score reflects the possibility of future osteoporotic fracture. Further, the National Osteoporosis Foundation guideline for treatment eligibility was changed to incorporate fracture history, $\mathrm{BMD}$, and 10-year fracture probability as per FRAX. Therefore, we analyzed the correlation between FRAX results and serum pentosidine levels to determine the significant risk factor of serum pentosidine in OVCF additionally. In this study, the 10year probability of major osteoporotic fracture, calculated using the FRAX, was significantly higher in the vertebral fracture group than in the non-fracture group. Moreover, there was a positive correlation between the serum pentosidine level and FRAX results $(r=0.35, p=0.02)$.

This study has certain limitations. First, the sample size was small, and the statistical power was weak. Second, there was nonhomogeneous demographic data between the vertebral fracture group and the non-fracture group. Third, there was no clarification for the cutoff pentosidine level for OVCF. However, despite these limitations, this study identified the potential areas for future research.

\section{Conclusions}

These results suggest that increased serum pentosidine level could be a potential risk factor for OVCF. However, to achieve more reliability, prospective large cohort studies are needed to investigate the true effect of the potential risk of high serum pentosidine level for OVCF in the future.

\section{Conflict of Interest}

No potential conflict of interest relevant to this article was reported.

\section{References}

1. NIH Consensus Development Panel on Osteoporosis Prevention, Diagnosis, and Therapy. Osteoporosis prevention, diagnosis, and therapy. JAMA 2001;285:785-95.

2. Kanis JA, Melton LJ 3rd, Christiansen C, Johnston CC, Khaltaev N. The diagnosis of osteoporosis. J Bone Miner Res 1994;9:1137-41. 
3. Baim S, Binkley N, Bilezikian JP, et al. Official positions of the International Society for Clinical Densitometry and executive summary of the 2007 ISCD Position Development Conference. J Clin Densitom 2008;11:75-91.

4. Simon LS. Osteoporosis. Clin Geriatr Med 2005;21:60329.

5. Viguet-Carrin S, Garnero P, Delmas PD. The role of collagen in bone strength. Osteoporos Int 2006;17:319-36.

6. Bailey AJ, Sims TJ, Ebbesen EN, Mansell JP, Thomsen JS, Mosekilde L. Age-related changes in the biochemical properties of human cancellous bone collagen: relationship to bone strength. Calcif Tissue Int 1999;65:203-10.

7. Wang X, Shen X, Li X, Agrawal CM. Age-related changes in the collagen network and toughness of bone. Bone 2002;31:1-7.

8. Saito M, Marumo K. Collagen cross-links as a determinant of bone quality: a possible explanation for bone fragility in aging, osteoporosis, and diabetes mellitus. Osteoporos Int 2010;21:195-214.

9. Oxlund H, Mosekilde L, Ortoft G. Reduced concentration of collagen reducible cross links in human trabecular bone with respect to age and osteoporosis. Bone 1996;19:479-84.

10. Vashishth D. The role of the collagen matrix in skeletal fragility. Curr Osteoporos Rep 2007;5:62-6.

11. Viguet-Carrin S, Roux JP, Arlot ME, et al. Contribution of the advanced glycation end product pentosidine and of maturation of type I collagen to compressive biomechanical properties of human lumbar vertebrae. Bone 2006;39:1073-9.

12. Yamamoto M, Yamaguchi T, Yamauchi M, Yano S, Sugimoto T. Serum pentosidine levels are positively associated with the presence of vertebral fractures in postmenopausal women with type 2 diabetes. J Clin Endocrinol Metab 2008;93:10139.

13. Shiraki M, Kuroda T, Tanaka S, Saito M, Fukunaga $M$, Nakamura T. Nonenzymatic collagen cross-links induced by glycoxidation (pentosidine) predicts vertebral fractures. J Bone Miner Metab 2008;26:93-100.

14. Kang HY, Yang KH, Kim YN, et al. Incidence and mortality of hip fracture among the elderly population in South Korea: a population-based study using the national health insurance claims data. BMC Public Health 2010;10:230.

15. Lippuner K, Johansson H, Kanis JA, Rizzoli R. Remaining lifetime and absolute 10-year probabilities of osteoporotic fracture in Swiss men and women. Osteoporos Int 2009;20:1131-40.

16. Urena P, De Vernejoul MC. Circulating biochemical markers of bone remodeling in uremic patients. Kidney Int 1999;55:2141-56.

17. Park C, Ha YC, Jang S, Jang S, Yoon HK, Lee YK. The incidence and residual lifetime risk of osteoporosis-related fractures in Korea. J Bone Miner Metab 2011;29:744-51.

18. So GY, Park KH, Yoon DH, Ryu JH, Choi YS. Feasibility of FRAX for prediction of osteoporotic vertebral fractures in Korea. Asian Spine J 2012;6:22-8.

19. Sell DR, Monnier VM. Structure elucidation of a senescence cross-link from human extracellular matrix: implication of pentoses in the aging process. J Biol Chem 1989;264:21597-602.

20. Saito M, Fujii K, Mori Y, Marumo K. Role of collagen enzymatic and glycation induced crosslinks as a determinant of bone quality in spontaneously diabetic WBN/Kob rats. Osteoporos Int 2006;17:1514-23.

21. Allen MR, Gineyts E, Leeming DJ, Burr DB, Delmas PD. Bisphosphonates alter trabecular bone collagen cross-linking and isomerization in beagle dog vertebra. Osteoporos Int 2008;19:329-37.

22. Hernandez CJ, Tang SY, Baumbach BM, et al. Trabecular microfracture and the influence of pyridinium and non-enzymatic glycation-mediated collagen cross-links. Bone 2005;37:825-32.

23. Odetti P, Rossi S, Monacelli F, et al. Advanced glycation end products and bone loss during aging. Ann N Y Acad Sci 2005;1043:710-7.

24. Sanguineti R, Storace D, Monacelli F, Federici A, Odetti P. Pentosidine effects on human osteoblasts in vitro. Ann N Y Acad Sci 2008;1126:16672.

25. Garnero P, Borel O, Gineyts E, et al. Extracellular post-translational modifications of collagen are major determinants of biomechanical properties of fetal bovine cortical bone. Bone 
2006;38:300-9.

26. Vashishth D, Gibson GJ, Khoury JI, Schaffler MB, Kimura J, Fyhrie DP. Influence of nonenzymatic glycation on biomechanical properties of cortical bone. Bone 2001;28:195-201.
27. Kanis JA, McCloskey EV, Johansson H, Oden A, Melton LJ 3rd, Khaltaev N. A reference standard for the description of osteoporosis. Bone 2008;42:467-75.

28. Kanis JA, Borgstrom F, De Laet C, et al. Assessment of fracture risk. Osteoporos Int 2005;16:581-9. 